\title{
ETERNAL DOMINATION: CRITICALITY AND REACHABILITY
}

\author{
William F. KLOSTERmeyer \\ School of Computing \\ University of North Florida \\ Jacksonville, FL 32224 USA \\ e-mail: wkloster@unf.edu \\ AND \\ Gary MacGillivray \\ Department of Mathematics and Statistics \\ University of Victoria, P.O. Box 3060 STN CSC \\ Victoria, BC, Canada V8W $3 R 4$ \\ e-mail: gmacgill@math.uvic.ca
}

\begin{abstract}
We show that for every minimum eternal dominating set, $D$, of a graph $G$ and every vertex $v \in D$, there is a sequence of attacks at the vertices of $G$ which can be defended in such a way that an eternal dominating set not containing $v$ is reached. The study of the stronger assertion that such a set can be reached after a single attack is defended leads to the study of graphs which are critical in the sense that deleting any vertex reduces the eternal domination number. Examples of these graphs and tight bounds on connectivity, edge-connectivity and diameter are given. It is also shown that there exist graphs in which deletion of any edge increases the eternal domination number, and graphs in which addition of any edge decreases the eternal domination number.
\end{abstract}

Keywords: dominating set, eternal dominating set, critical graphs.

2010 Mathematics Subject Classification: 05C69.

\section{REFERENCES}

[1] R.C. Brigham, P.Z. Chinn and R.D. Dutton, Vertex domination-critical graphs, Networks 18 (1988) 173-179. doi:10.1002/net.3230180304 
[2] A.P. Burger, E.J. Cockayne, W.R. Gründlingh, C.M. Mynhardt, J.H. van Vuuren and W. Winterbach, Infinite order domination in graph, J. Combin. Math. Combin. Comput. 50 (2004) 179-194.

[3] W. Goddard, S.M. Hedetniemi and S.T. Hedetniemi, Eternal security in graphs, J. Combin. Math. Combin. Comput. 52 (2005) 169-180.

[4] T.W. Haynes, S.T. Hedetniemi and P.J. Slater, Fundamentals of Domination in Graphs (Marcel Dekker, New York, 1998).

[5] G. Fricke, S.M. Hedetniemi, S.T. Hedetniemi and K.R. Hutson, $\gamma$-graphs of graphs, Discuss. Math. Graph Theory 31 (2011) 517-531.

doi:10.7151/dmgt.1562

[6] J. Fulman, D. Hanson and G. MacGillivray, Vertex domination-critical graphs, Networks 25 (1995) 41-43.

[7] R. Haas and K. Seyffarth, The k-dominating graph, Graphs Combin. 30 (2014) 609-617. doi:10.1007/s00373-013-1302-3

[8] W. Klostermeyer, M. Lawrence and G. MacGillivray, Dynamic dominating sets: the eviction model for eternal domination, J. Combin. Math. Combin. Comput. (2016), to appear.

[9] W. Klostermeyer and G. MacGillivray, Eternal dominating sets in graphs, J. Combin. Math. Combin. Comput. 68 (2009) 97-111.

[10] W. Klostermeyer and C.M. Mynhardt, Protecting a graph with mobile guards, Appl. Anal. Discrete Math. (2016), to appear.

Received 10 August 2015

Revised 16 February 2016

Accepted 16 February 2016 